\title{
Special issue on quantum walks
}

\author{
Yutaka Shikano
}

Received: 8 June 2012 / Accepted: 4 July 2012 / Published online: 15 July 2012

(C) Springer Science+Business Media, LLC 2012

Understanding quantum dynamical features seems to be needed to open the next step of quantum information science. One strong candidate to understand quantum dynamical phenomena is a quantum walk. Quantum walks are defined as the quantummechanical analogue of the classical random walk. However, those are not the quantization of the classical random walks. Like the classical random walks, there may be many applications of the quantum walks. Therefore, the community of the quantum walk is an interdisciplinary field from mathematics to the experimental physics. The quantum walks have two types; the discrete-time and the continuous-time quantum walks. Both common and unique features are the followings; (i) the ballistic transport system, which corresponds to quantum transportation and (ii) non-normal probability distribution under the uniform system, and (iii) the Anderson-like localization. By combining these features, quantum walks show an intriguing phenomena in mathematics, physics, and computer science. The community of quantum walks is gradually increased. They held some workshops, e.g., at Tokyo Institute of Technology, Tokyo, Japan in 2011 (cancellation due to the Japan big earthquakes and aftermath) and at Instituto de Fisica Corpuscular, Valencia, Spain in 2011. A workshop will be held at the Institute for Molecular Science, Okazaki, Japan on November, 2012 to collect the quantum walkers. Many scientists have a potential to study the quantum walks.

The aim of this special issue is to know what is the role of quantum walks for the other potentially related fields and to reveal many varieties of unique aspects and open problems on the quantum walks. Therefore, we have prepared three comprehensive reviews from mathematics, theoretical physics, and quantum information, and seven contributed articles from theoretical viewpoints. I hope that the quantum walks

\footnotetext{
Y. Shikano $(\varangle)$

Institute for Molecular Science, 38 Nishigo-Naka, Myodaiji, Okazaki 444-8585, Japan

e-mail: yshikano@ims.ac.jp
} 
will become the key tools to understand quantum dynamical phenomena in the near future.

Finally, I would like to thank Howard Bradt for giving me the great opportunity to publish the special issue on quantum walks and Sithara Shenoy for the editorial supports. 